\title{
Dynamic changes in fat oxidation in human primary myocytes mirror metabolic characteristics of the donor
}

\author{
Barbara Ukropcova, Michele McNeil, Olga Sereda, Lilian de Jonge, Hui Xie, \\ George A. Bray, and Steven R. Smith \\ Pennington Biomedical Research Center, Baton Rouge, Louisiana, USA
}

\begin{abstract}
Metabolic flexibility of skeletal muscle, that is, the preference for fat oxidation (FOx) during fasting and for carbohydrate oxidation in response to insulin, is decreased during insulin resistance. The aim of this study was to test the hypothesis that the capacity of myotubes to oxidize fat in vitro reflects the donor's metabolic characteristics. Insulin sensitivity (IS) and metabolic flexibility of $\mathbf{1 6}$ healthy, young male subjects was determined by euglycemic hyperinsulinemic clamp. Muscle samples were obtained from vastus lateralis, cultured, and differentiated into myotubes. In human myotubes in vitro, we measured suppressibility (glucose suppression of FOx) and adaptability (an increase in FOx in the presence of high palmitate concentration). We termed these dynamic changes in FOx metabolic switching. In vivo, metabolic flexibility was positively correlated with IS and maximal oxygen uptake and inversely correlated with percent body fat. In vitro suppressibility was inversely correlated with IS and metabolic flexibility and positively correlated with body fat and fasting FFA levels. Adaptability was negatively associated with percent body fat and fasting insulin and positively correlated with IS and metabolic flexibility. The interindividual variability in metabolic phenotypes was preserved in human myotubes separated from their neuroendocrine environment, which supports the hypothesis that metabolic switching is an intrinsic property of skeletal muscle.
\end{abstract}

\section{Introduction}

Obesity and type 2 diabetes are characterized by an increase in body fat, a decrease in insulin-stimulated glucose disposal, and disturbances of oxidative metabolism. Skeletal muscle, the organ responsible for the majority of insulin-stimulated glucose uptake, has a decreased oxidative capacity in obesity and diabetes (1-3) and after weight loss (4-7). Furthermore, Zurlo (8) and others (9-11) showed that decreased fat oxidation (FOx) (represented by a higher respiratory quotient [RQ], the ratio of $\mathrm{CO}_{2}$ produced to $\mathrm{O}_{2}$ consumed) is a predictor of weight gain, which suggests the importance of early defects in FOx for future development of obesity and diabetes. Defects in skeletal muscle oxidative capacity and fat metabolism are highly correlated with insulin sensitivity (IS) $(1,5,12)$ and are believed to contribute to the pathogenesis of insulin resistance $(13,14)$.

A high-fat diet (HFD) challenges the oxidative machinery of skeletal muscle. Substantial variability exists in the ability of individuals to adapt to HFD by increasing FOx (15). An imbalance between fat intake and FOx results in a positive fat balance (15). Decreased adaptation to HFD has been observed in restrained eaters (16), formerly obese $(17,18)$ and obese individuals $(19)$, and individuals with a family history of obesity (20). The latter study points toward a possible genetic basis for reduced FOx. Attenuated adaptation to HFD might represent yet another feature of "metabolic inflexibility," the impaired ability of skeletal muscle to switch from carbohydrate to fat oxidation during fasting and from fat to car-

Nonstandard abbreviations used: ASP, acid-soluble product; FOx, fat oxidation; HFD, high-fat diet; IS, insulin sensitivity; LBM, lean body mass; $\triangle R Q$ (clamp), change in RQ during a clamp; RQ, respiratory quotient; TOx, total palmitate oxidation; $\mathrm{VO}_{2} \max$, maximal oxygen uptake.

Conflict of interest: The authors have declared that no conflict of interest exists.

Citation for this article: J. Clin. Invest. 115:1934-1941 (2005).

doi:10.1172/JCI24332. bohydrate oxidation in response to insulin $(14,21)$. The question is, what plays the major role in the handling of a HFD challenge - the neuroendocrine environment, e.g., insulin, leptin, adiponectin, or the capacity of skeletal muscle to oxidize fatty acids?

Obese and lean individuals differ in their response to hyperinsulinemia. Healthy lean individuals use lipids as a main source of fuel under fasting conditions, and they readily switch to carbohydrate oxidation in response to insulin. On the contrary, the inflexible muscle of an insulin-resistant individual is characterized by lower fasting lipid utilization and does not switch to carbohydrate oxidation in response to insulin $(5,14,22,23)$. Kelley and Mandarino pointed out this loss of the "normal" fluctuations in carbohydrate and FOx in insulin-resistant states and named it metabolic inflexibility (14). The lower fasting FOx in insulin resistance might be explained simply by the decreased capacity of skeletal muscle to oxidize fat (1-3). Another possibility is suppression of FOx by glucose, known as reverse Randle cycle (24-26).

In obesity, skeletal muscle could be an innocent bystander, insulin resistant and inflexible because of neuroendocrine and environmental factors. There is good evidence for defects in the endocrine milieu (27-29). Impaired secretion of insulin, a major regulator of substrate utilization in vivo, could contribute to the inflexible phenotype (13). High fatty acid concentrations result in insulin resistance in a matter of minutes (30-33). A number of studies point out detrimental effects of a HFD, which lead to the accumulation of lipids and their intermediates (diacylglycerols, ceramides, long-chain acyl-CoAs) associated with insulin resistance (34-38). Alternatively, metabolic inflexibility and insulin resistance might be phenotypes intrinsic to the muscle cells themselves, retained in vitro by either genetic, epigenetic, or other, yet-unknown, mechanisms. Muscle cells from insulin-resistant individuals remain insulin resistant in vitro (39-42), which lends strong support to the hypothesis that 
Table 1

Clinical characteristics of the study population before the HFD

\begin{tabular}{lcc} 
Phenotype & Mean \pm SD & Range \\
Ethnicity (white/Asian) & $12 / 4$ & \\
Age $(\mathrm{yr})$ & $22.4 \pm 3.6$ & $18-29$ \\
Body weight $(\mathrm{kg})$ & $78.8 \pm 14.2$ & $59.2-105.8$ \\
$\mathrm{BMI}\left(\mathrm{kg} / \mathrm{m}^{2}\right)$ & $25.8 \pm 4.2$ & $20.1-32.9$ \\
Fat mass $(\mathrm{kg})$ & $15.89 \pm 6.8$ & $7.27-26.89$ \\
$\%$ Body fat & $19.7 \pm 6.8$ & $10.7-32.3$ \\
Waist circumference $(\mathrm{cm})$ & $82.5 \pm 9.7$ & $72.9-98.5$ \\
$\mathrm{VO}_{2} \mathrm{max}(\mathrm{ml} / \mathrm{kg} / \mathrm{min})$ & $41.6 \pm 7.7$ & $31.8-54.4$ \\
$\mathrm{IS}(\mathrm{mg} / \mathrm{kg} \mathrm{LBM} / \mathrm{min})$ & $10.8 \pm 4.0$ & $5.8-18.8$ \\
Fasting RQ $(\mathrm{clamp})$ & $0.837 \pm 0.03$ & $0.78-0.86$ \\
$\Delta \mathrm{RQ}(\mathrm{clamp})$ & $0.074 \pm 0.037$ & $0.03-0.14$ \\
$24-\mathrm{h} \mathrm{RQ} \mathrm{STD}$ & $0.865 \pm 0.004$ & $0.85-0.87$ \\
Fasting glucose $(\mathrm{mg} / \mathrm{dl})$ & $82.0 \pm 5.6$ & $70-90$ \\
Fasting insulin $(\mu \mathrm{U} / \mathrm{ml})$ & $7.86 \pm 4.9$ & $2.3-19.4$ \\
Fasting triglycerides $(\mathrm{mg} / \mathrm{dl})$ & $111.3 \pm 92.2$ & $33-328$ \\
Fasting FFAs $(\mathrm{mmol} / \mathrm{l})$ & $0.41 \pm 0.12$ & $0.23-0.66$ \\
\hline
\end{tabular}

$\mathrm{RQ}$ was calculated as the ratio of $\mathrm{CO}_{2}$ production to $\mathrm{O}_{2}$ consumption; IS, the glucose disposal rate, measured by clamp; $\Delta R Q$ (clamp) $=R Q$ at the end of the insulin-stimulated glucose infusion - fasting RQ; 24-h RQ STD, 24-hour respiratory quotient measured in patients in a wholeroom calorimeter on a standard diet (35\% fat, $16 \%$ protein, $49 \%$ carbohydrate), adjusted for energy balance.

skeletal muscle insulin resistance is a primary disorder of skeletal muscle and has a genetic or epigenetic origin. In contrast, much less evidence exists regarding defects in muscle FOx ex vivo (43-45).

The purpose of this study was to test the hypothesis that metabolic switching, represented by dynamic changes in FOx in vitro, is an intrinsic characteristic of skeletal muscle cells, independent of influences of the whole-body environment. We cultured muscle satellite cells from individuals with differing clinical characteristics in a standardized, insulin-free medium and measured 2 parameters of metabolic switching in vitro: (a) suppressibility - glucose suppression of FOx, a measure of fuel preference in the absence of insulin; and (b) adaptability - the increase in FOx in the presence of high palmitate concentration. We related these responses of myotubes in vitro to the clinical phenotypes of their donors.

\section{Results}

The characteristics of the 16 men in this study are presented in Table 1. In general, they were young and healthy with a broad range of BMI $\left(20.1-32.9 \mathrm{~kg} / \mathrm{m}^{2}\right)$, fat mass $(7.27-26.89 \mathrm{~kg})$, and IS (5.8-18.8 mg/kg lean body mass/min [mg/kg LBM/min]).

Relationship between metabolic flexibility and IS in vivo. As expected, IS, measured by euglycemic hyperinsulinemic clamp (clamp), correlated negatively with percentage body fat $(r=-0.60 ; P=0.008)$ and positively with maximal oxygen uptake $\left(\mathrm{VO}_{2} \mathrm{max}\right)(r=0.59$; $P=0.015)$, which confirms the well-documented relationship among body fat mass, fitness, and IS (46-48). A negative correlation was observed between IS and fasting levels of both insulin $(r=-0.65 ; P=0.0003)$ and FFAs $(r=-0.81 ; P<0.0001)$. Fasting RQ during the clamp, a parameter of fasting fuel partitioning, was related neither to IS $(P=0.80)$ nor to anthropometric characteristics of subjects (percent body fat; $P=0.60$ ). Metabolic flexibility, defined as the change in RQ during a clamp [ $\Delta \mathrm{RQ}$ (clamp)], was negatively correlated with percent body fat and fasting FFA levels, which indicated a lower capacity for metabolic switching between fuels in subjects with higher fat mass and increased FFA levels (Table 2). On the other hand, $\Delta R Q$ (clamp) correlated positively with IS and $\mathrm{VO}_{2} \max$, which supports the existence of a relationship between metabolic flexibility and both IS and aerobic fitness, respectively (Table 2). Taken together, these data demonstrate that defects in metabolic flexibility are present in healthy, young men and mirror the defects seen in older subjects with obesity and type 2 diabetes.

In vitro glucose suppressibility. An increase in the extracellular glucose concentration in vitro resulted in a decrease of FOx as indicated by levels of both ${ }^{14} \mathrm{CO}_{2}$ and ${ }^{14} \mathrm{C}$-intermediate metabolites of $\mathrm{FOx}$ (acidsoluble products [ASPs] $)\left(r=-0.96, P<0.0001\right.$ for ${ }^{14} \mathrm{CO}_{2} ; r=-0.95$, $P<0.0001$ for ASPs; data not shown). A positive relationship was observed between percent suppression of ${ }^{14} \mathrm{CO}_{2}$ by glucose (\% suppression $=[1-(\mathrm{FOx}$ at $5 \mathrm{mM}$ glucose $/ \mathrm{FOx}$ at $0 \mathrm{mM}$ glucose $)] \times$ $100)$ and percent suppression of total palmitate oxidation (TOx) by glucose $\left(\mathrm{TOx}={ }^{14} \mathrm{CO}_{2}+{ }^{14} \mathrm{C}\right.$-ASPs $)(r=0.51 ; P=0.03)$.

In vitro metabolic adaptability. Increasing palmitate concentration in vitro produced an increase in ${ }^{14} \mathrm{CO}_{2}$ and ASP production $(r=0.998 ; P<0.0001$; Figure 1B). However, in some subjects, the maximal rate of FOx (reflected by ASP production) flattened out at $100 \mu \mathrm{M}$ palmitate, which suggests an inability to further oxidize fatty acids. An inverse relationship was observed between basal FOx (at $1 \mu \mathrm{Ci} / \mathrm{ml}{ }^{14} \mathrm{C}$-palmitate with $0 \mu \mathrm{M}$ cold palmitate) and the fold increase in FOx $(r=-0.51 ; P=0.025)$. For both assays, ${ }^{14} \mathrm{CO}_{2}$ represented $10-30 \%$ of the total FOx, which indicates that the majority of palmitate was processed through $\beta$-oxidation to intermediate metabolites (ASPs). No significant relationship was observed between fold increase in ${ }^{14} \mathrm{CO}_{2}$ (in the in vitro adaptability assay) and percent suppression of ${ }^{14} \mathrm{CO}_{2}$ (in the in vitro suppressibility assay), when all 16 subjects were used for the analysis. However, a significant correlation was present after 1 subject was eliminated with a value greater than 3 SDs away from the mean of the remaining subjects $(r=-0.46 ; P=0.048 ; n=15)$. This finding supported the hypothesis that subjects with greater capacity to increase FOx when acutely exposed to high levels of palmitate would maintain relatively high FOx when exposed to glucose, in an insulin-free environment. For fold change of TOx, no significant relationship was found between the results of the 2 assays. The results of both in vitro assays are summarized in Table 3.

Reproducibility. We next validated the reproducibility of FOx assays by demonstrating the reliability of FOx measurement. Both FOx assays were performed on 2 different occasions, in a random subset of the whole population ( $n=7$ or 8 ). Results for both parameters of metabolic switching, suppressibility and adaptability, were highly reproducible (Supplemental Figure 1; supplemental material available online with this article; doi:10.1172/JCI24332DS1).

\section{Table 2}

Metabolic flexibility correlates with clinical characteristics of subjects in vivo

\begin{tabular}{lcll}
\hline $\begin{array}{l}\text { Clinical } \\
\text { characteristic }\end{array}$ & $\begin{array}{c}\text { Fasting RQ } \\
\text { (clamp) }\end{array}$ & $\begin{array}{c}\Delta \mathbf{R Q} \\
\text { (clamp) }\end{array}$ & 24-h RQ STD \\
Body fat $(\%)$ & $r=0.1$ & $r=-0.51^{\mathrm{A}}$ & $r=0.27$ \\
$\mathrm{IS}(\mathrm{mg} / \mathrm{kg} \mathrm{LBM} / \mathrm{min})$ & $r=-0.06$ & $r=0.90^{\mathrm{B}}$ & $r=-0.17$ \\
$\mathrm{VO}_{2} \mathrm{max}(\mathrm{ml} / \mathrm{kg} / \mathrm{min})$ & $r=-0.08$ & $r=0.59^{\mathrm{A}}$ & $r=-0.58^{\mathrm{A}}$ \\
$\mathrm{FFA}(\mathrm{mmol} / \mathrm{l})$ & $r=0.28$ & $r=-0.74^{\mathrm{B}}$ & $r=-0.21$ \\
\hline
\end{tabular}

$n=16 .{ }^{A} P<0.05 ;{ }^{B} P<0.005$. 

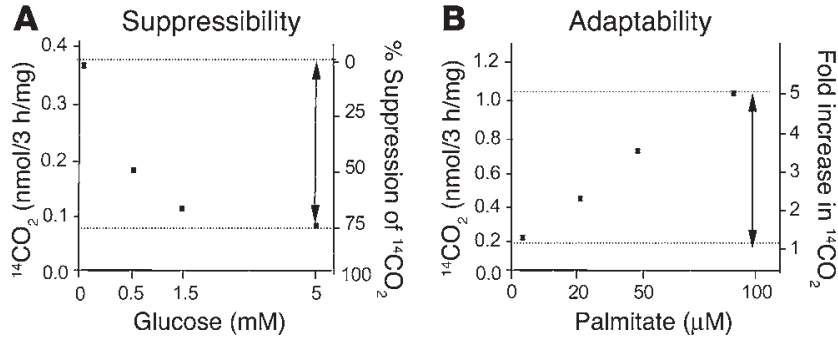

Figure 1

Characteristics of in vitro metabolic switching assays. (A) Dose response of $\mathrm{FOx}$ (measured by ${ }^{14} \mathrm{CO}_{2}$ production) to an increasing glucose concentration (suppressibility). (B) Dose response of FOx (measured by ${ }^{14} \mathrm{CO}_{2}$ production) to an increasing palmitate concentration (adaptability). Arrows indicate a dynamic change in FOx, representing an in vitro correlate to the clinical phenotype. Muscle cells were grown and differentiated into myotubes in 24-well plates. FOx assays were performed after 5 days of differentiation. Myotubes were incubated for 3 hours in serum-free media with increasing concentrations of glucose or palmitate. Assays were performed in duplicate, and data were normalized to protein content. Data are from 1 representative experiment.

Glucose suppressibility of FOx is an intrinsic characteristic of muscle cells. The ability of glucose to suppress FOx in myotubes in vitro is represented by the percent suppression of ${ }^{14} \mathrm{CO}_{2}$ production. It was inversely correlated with in vivo metabolic flexibility [as indicated by $\triangle \mathrm{RQ}($ clamp)] $(r=-0.61 ; P=0.007)$ and IS (as measured by clamp) $(r=-0.60 ; P=0.007)$ (Table 4 and Figure $2, \mathrm{~A}$ and $\mathrm{B}$ ), which supports the hypothesis that metabolic phenotypes persist in vitro and reflect the in vivo pattern of response of the muscle cell. The inverse correlation between suppressibility and IS remained significant after IS was adjusted for fat mass $(r=-0.47$; $P=0.04)$. Similarly, we found a correlation between the suppressibility of FOx by glucose (percent suppression of ${ }^{14} \mathrm{CO}_{2}$ ) in vitro and percent body fat $(r=0.56 ; P=0.02)$ and fasting FFA levels $(r=0.58 ; P=0.01$; Figure $2, \mathrm{C}$ and $\mathrm{D})$ in vivo. However, these in vitro-in vivo relationships were only observed for $\mathrm{CO}_{2}$, not for total FOx $(P=0.5$ for percent body fat; $P=0.1$ for FFA levels $)$, which suggests a dominant role of $\mathrm{CO}_{2}$ (via tricarboxylic acid cycle) over intermediate metabolites of FOx (via $\beta$-oxidation). Neither percentage suppression of ${ }^{14} \mathrm{CO}_{2}$ nor percentage suppression of TOx correlated with fasting RQ (measured by clamp) or 24hour RQ (measured by indirect calorimetry) in vivo. A summary of in vivo-in vitro relationships is shown in Table 4.

Metabolic adaptability is an intrinsic characteristic of muscle cells. The ability of cells to increase FOx when acutely exposed to a high palmitate concentration (fold increase in ${ }^{14} \mathrm{CO}_{2}$ ) is a measure of adaptability of muscle cells. Fold increase in ${ }^{14} \mathrm{CO}_{2}$ was positively correlated with in vivo metabolic flexibility [as indicated by $\triangle \mathrm{RQ}$ (clamp)] $(r=0.53$; $P=0.035$; Figure 3A), IS (as measured by clamp) $(r=0.60 ; P=0.015$; Figure $3 \mathrm{~B})$ and aerobic capacity $\left(\mathrm{VO}_{2} \max \right)$

Table 3

Results of in vitro F0x assays $(r=0.70 ; P=0.002$; Figure $3 C)$, respectively. However, the relationship between adaptability and IS was blunted after IS was adjusted for body fat $(P=0.58)$. A negative relationship was observed between body fat $(r=-0.62 ; P=0.01$, Figure $3 \mathrm{D})$ and a proxy of abdominal fatness, waist circumference $(r=-0.4 ; P=0.01)$. A highly significant correlation was observed between the fold increase in ${ }^{14} \mathrm{CO}_{2}$ in vitro and fasting insulin in vivo $(r=-0.81 ; P=0.0002$; Figure $3 \mathrm{E})$. Weaker relationships were found between the fold increase in TOx in vitro and level of body fat $(r=-0.37 ; P=0.1)$. The relationships between fold increase in TOx and waist circumference or fasting insulin level were statistically significant $(r=-0.34, P=0.02$ and $r=-0.55$, $P=0.027$, respectively). As was the case with the in vitro glucose suppressibility assay, $\mathrm{CO}_{2}$ production appeared to be a better correlate with the clinical phenotypes than was ASP production. A summary of in vivo-in vitro relationships is shown in Table 4.

\section{Discussion}

Obesity is associated with insulin resistance, impaired oxidative capacity, and metabolic inflexibility of skeletal muscle. Kelley et al. defined metabolic inflexibility as a low fasting FOx and a decreased ability to switch from fat to carbohydrate oxidation in response to insulin (14). The key question is whether inflexibility is a byproduct of our obesigenic environment or a phenotype intrinsic to the skeletal muscle. To address this question, we used primary human skeletal muscle cells, cultured for up to 5 weeks in a standardized medium, isolated from other influences. This model is ideal for studying interindividual differences of metabolism intrinsic to the skeletal muscle, i.e., independent of the influences of the whole-body environment, and retained in vitro by genetic or epigenetic mechanisms.

The principal finding from this study is that metabolic switching, represented by dynamic changes in FOx in muscle cells, reflects the metabolic characteristics of the cells' donor. Suppressibility, representing a glucose suppression of FOx in myotubes, was inversely associated with metabolic flexibility and IS and positively correlated with body fat and fasting FFA levels. Adaptability, the capacity of myotubes to increase FOx with a high palmitate concentration, was positively correlated with metabolic flexibility, IS, and aerobic capacity and inversely associated with body fat and fasting insulin levels (Figure 4). The relatively small number of subjects with a broad range of metabolic phenotypes limited categorical analysis of the data across clinical phenotypes of IS, body fat, etc. However, our observations based on a cross-sectional

\section{F0x assay}

Suppression of FOx with increasing glucose concentration (nmol/3 $\mathrm{h} / \mathrm{mg}$ protein)

In vitro suppressibility

Increase of FOx with increasing palmitate concentration ( $\mathrm{nmol} / 3 \mathrm{~h} / \mathrm{mg}$ protein) In vitro adaptability

$\begin{array}{ccc}\text { Fox rate } & \text { Mean } \pm \text { SD } & \text { Range } \\ \text { Maximal }{ }^{14} \mathrm{CO}_{2} & 0.57 \pm 0.43 & 0.15-1.96 \\ \text { Suppressed }{ }^{14} \mathrm{CO}_{2} & 0.24 \pm 0.18 & 0.07-0.82 \\ \% \text { Suppression of }{ }^{14} \mathrm{CO}_{2} & 58.72 \pm 10.18 & 40.0-81.37 \\ { }^{14} \mathrm{CO}_{2} \text { at basal palmitate } & 0.33 \pm 0.25 & 0.15-0.71 \\ { }^{14} \mathrm{CO}_{2} \text { at } 100 \mu \mathrm{M} \text { palmitate } & 1.27 \pm 0.7 & 0.67-3.37 \\ \text { Fold increase in }{ }^{14} \mathrm{CO}_{2} & 3.19 \pm 0.76 & 2.99-4.87\end{array}$

In vitro suppressibility, suppression of ${ }^{14} \mathrm{CO}_{2}$ production by glucose; \% Suppression of ${ }^{14} \mathrm{CO}_{2}=[1-(\mathrm{FOx}$ at 5 $\mathrm{mM}$ glucose / FOx at $0 \mathrm{mM}$ glucose) $] \times 100$. Maximal ${ }^{14} \mathrm{CO}_{2}$ was measured at $20 \mu \mathrm{M}$ palmitate, 0 mM glucose; suppressed ${ }^{14} \mathrm{CO}_{2}$ was measured at $20 \mathrm{uM}$ palmitate, $5 \mathrm{mM}$ glucose. In vitro adaptability, an increase in ${ }^{14} \mathrm{CO}_{2}$ production with an acute exposure to high [palmitate]); fold increase in ${ }^{14} \mathrm{CO}_{2}={ }^{14} \mathrm{CO}_{2}$ at $100 \mu \mathrm{M}$ palmitate / ${ }^{14} \mathrm{CO}_{2}$ at basal palmitate; basal palmitate, $1 \mu \mathrm{Ci} / \mathrm{ml}\left[{ }^{14} \mathrm{C}\right]$ palmitate, $0 \mu \mathrm{M}$ cold palmitate. For all assays, $1 \mu \mathrm{Ci} / \mathrm{ml}$ $\left[1-{ }^{14} \mathrm{C}\right]$ palmitate was used. Assays were performed in duplicate, and data were normalized to protein content. 
Table 4

Parameters of metabolic switching in vitro correlate with clinical phenotypes of subjects

\begin{tabular}{|c|c|c|c|c|c|c|c|c|c|c|}
\hline \multirow{2}{*}{\multicolumn{2}{|c|}{$\begin{array}{l}\text { In vitro parameters } \\
\text { of metabolic switching }\end{array}$}} & \multicolumn{3}{|c|}{ Metabolic flexibility in vivo } & \multicolumn{6}{|c|}{ Clinical characteristics of subjects } \\
\hline & & $\begin{array}{l}\text { Fasting RQ } \\
\text { (clamp) }\end{array}$ & $\begin{array}{c}\Delta R Q \\
\text { (clamp) }\end{array}$ & $\begin{array}{c}\text { 24-h RQ } \\
\text { STD }\end{array}$ & $\begin{array}{c}\text { IS } \\
(\mathrm{mg} / \mathrm{kg} / \\
\mathrm{min})\end{array}$ & $\begin{array}{l}\text { Waist } \\
\text { circum. } \\
\text { (cm) }\end{array}$ & $\begin{array}{c}\text { Body } \\
\text { fat } \\
(\%)\end{array}$ & $\begin{array}{c}\mathrm{VO}_{2} \max \\
(\mathrm{ml} / \mathrm{kg} / \\
\mathrm{min})\end{array}$ & $\begin{array}{c}\text { Fasting } \\
\text { FFA } \\
(\mathrm{mmol} / \mathrm{l})\end{array}$ & $\begin{array}{c}\text { Fasting } \\
\text { insulin } \\
(\mu \mathrm{U} / \mathrm{ml})\end{array}$ \\
\hline Adaptability & $\begin{array}{l}\text { Fold increase in } \\
{ }^{14} \mathrm{CO}_{2} \text { with palmitate }\end{array}$ & $r=-0.25$ & $r=0.53^{\mathrm{A}}$ & $r=-0.30$ & $r=0.60^{A}$ & $r=-0.39 A$ & $r=-0.62^{A}$ & $r=0.70^{B}$ & $r=-0.41$ & $r=-0.81^{B}$ \\
\hline Suppressibility & $\begin{array}{l}\% \text { Suppression of } \\
{ }^{14} \mathrm{CO}_{2} \text { by glucose }\end{array}$ & $r=0.12$ & $r=-0.61^{A}$ & $r=-0.05$ & $r=-0.60^{A}$ & $r=0.11$ & $r=0.56^{A}$ & $r=-0.44$ & $r=0.58^{A}$ & $r=0.35$ \\
\hline
\end{tabular}

Basal palmitate, $0 \mu \mathrm{M}$ cold palmitate, $1 \mu \mathrm{Ci} / \mathrm{ml}$ labeled palmitate; \% Suppression of ${ }^{14} \mathrm{CO}_{2}=[1-(\mathrm{FOx}$ at $5 \mathrm{mM}$ glucose $/ \mathrm{FOx}$ at $0 \mathrm{mM}$ glucose) $\times$ × 100 ; For all assays, $1 \mu \mathrm{Ci} / \mathrm{ml}\left[1-{ }^{14} \mathrm{C}\right]$ palmitate was used. Assays were performed in duplicate, and data were normalized to protein content. Data for fold increase in ${ }^{14} \mathrm{CO}_{2}$ have been adjusted for basal ${ }^{14} \mathrm{CO}_{2}$ production. $n=16$. ${ }^{A} P<0.05$, ${ }^{\mathrm{B}} P<0.005$. Circum, circumference.

analysis clearly demonstrate the relationship between clinical phenotypes and dynamic changes in FOx in cultured myotubes, which supports the hypothesis that metabolic switching is, at least to a certain extent, an intrinsic characteristic of skeletal muscle. Our data does not exclude the potentially powerful modifying effects of "extrinsic" influences - environmental or neuroendocrine - on metabolic switching. As was shown previously, the metabolic phenotype can be substantially influenced by external factors such as weight loss (5), thiazolidenedione treatment (49), and exercise (50). Whether these interventions would have an effect on metabolic switching in vitro is a question requiring further study.

In vivo, we observed the expected positive relationship between metabolic flexibility and both IS and aerobic fitness. This is in agreement with the previous findings of Kelley et al., who showed that obese and type 2 diabetic individuals have a defect in the ability to switch between fuels in response to insulin, indicating the state of metabolic inflexibility $(5,14)$.

Our in vitro results demonstrate that glucose suppresses FOx in the absence of insulin. This reverse Randle cycle was demonstrated to operate in myocytes (51) and animal models (52), as well as in lean, healthy individuals (32) and type 2 diabetics (22). Importantly, we found substantial interindividual differences in the magnitude of glucose suppression of FOx in vitro. This indicates that factors other than hyperglycemia, such as basal glucose uptake $(53,54)$ and mitochondrial

\section{Figure 2}

In vitro suppressibility is an intrinsic characteristic of muscle cells. The potential of glucose to suppress $\mathrm{FOx}\left(\mathrm{CO}_{2}\right)$ in vitro (\% suppression of ${ }^{14} \mathrm{CO}_{2}=[1-(\mathrm{FOx}$ at $5 \mathrm{mM}$ glucose $/ \mathrm{FOx}$ at $0 \mathrm{mM}$ glucose) $] \times 100$ ) is correlated with in vivo metabolic flexibility [as indicated by $\triangle R Q$ (clamp), an insulin-stimulated change in $R Q$, measured by indirect calorimetry during the clamp] (A); in vivo IS represented by glucose disposal rate, measured by clamp (B); percent body fat, measured by dual energy $\mathrm{X}$-ray absorptiometry $(\mathbf{C})$; and in vivo fasting FFA levels (D). Muscle cells from 16 individuals were grown and differentiated into myotubes in 24-well plates. Myotubes were preincubated in glucose- and serum-free medium and incubated for 3 hours with $1 \mu \mathrm{Ci} / \mathrm{ml}{ }^{14} \mathrm{C}$-palmitate, without glucose (to measure maximal $\mathrm{FOx}$ ) or with $5 \mathrm{mM}$ glucose (to measure suppressed FOx). After incubation, ${ }^{14} \mathrm{CO}_{2}$ and ${ }^{14} \mathrm{C}$-intermediate metabolites of FOx were determined. Assays were performed in duplicates and data were normalized to protein content. number and function $(3,55,56)$, might play an important role in the regulation of insulin-independent substrate utilization in muscle cells. However, the mechanisms by which glucose elicits a differential effect on FOx in vitro will require further study.

Adaptability represents an increase of FOx in myotubes in response to an increased fatty acid concentration. Previous studies have shown changes in substrate partitioning and a decrease in oxidative capacity in formerly obese, obese, and diabetic individuals $(5-7,57,58)$. We showed that there are individual differences in adaptation to a HFD in vivo (15). However, much less information is available about whether differences in the capacity to oxidize fat are preserved in vitro (43-45). In our model, short-term exposure of myotubes to increased palmitate levels enhanced FOx 3- to 5-fold, the magnitude of response correlating with clinical phenotype. Adaptability is the result of a concert of events, including uptake and transport of fatty acids, fuel sensor systems, and cellular oxidative machinery (59-63). The importance of these mechanisms for the observed in vitro phenotype remains to be determined.

Previous studies found defects in the absolute rate of FOx only in skeletal muscle cells or muscle homogenates from individuals
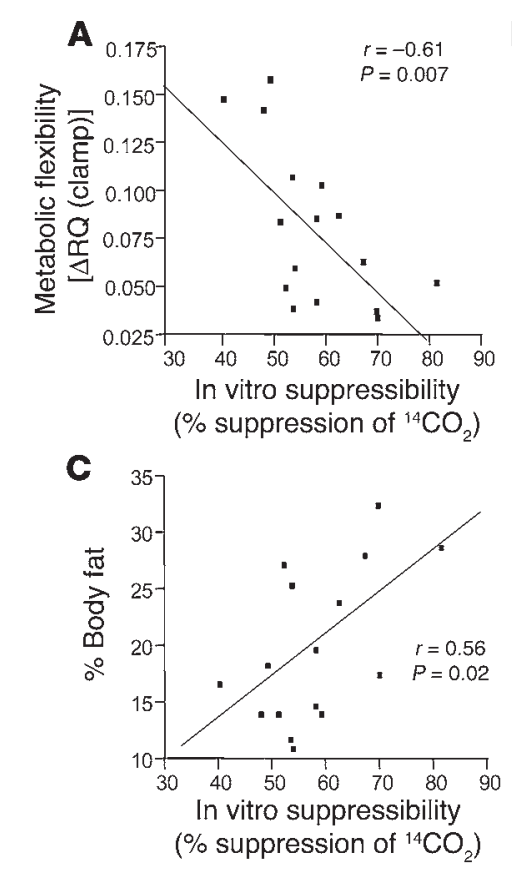
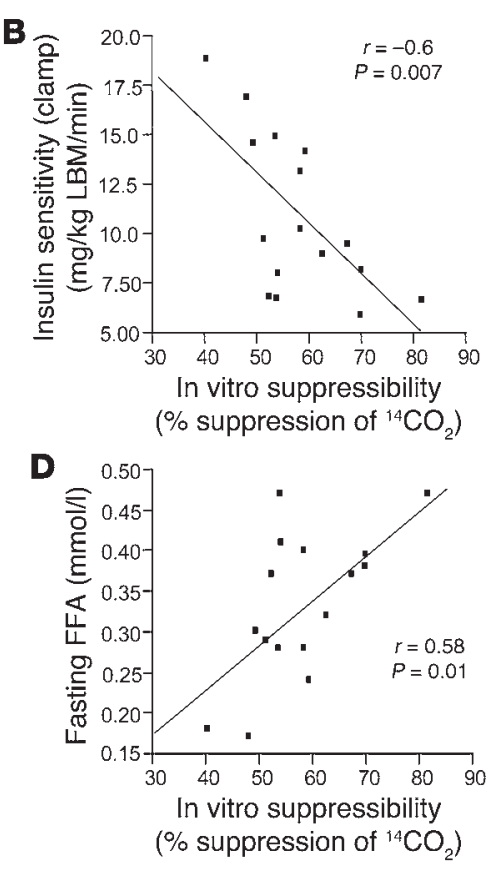

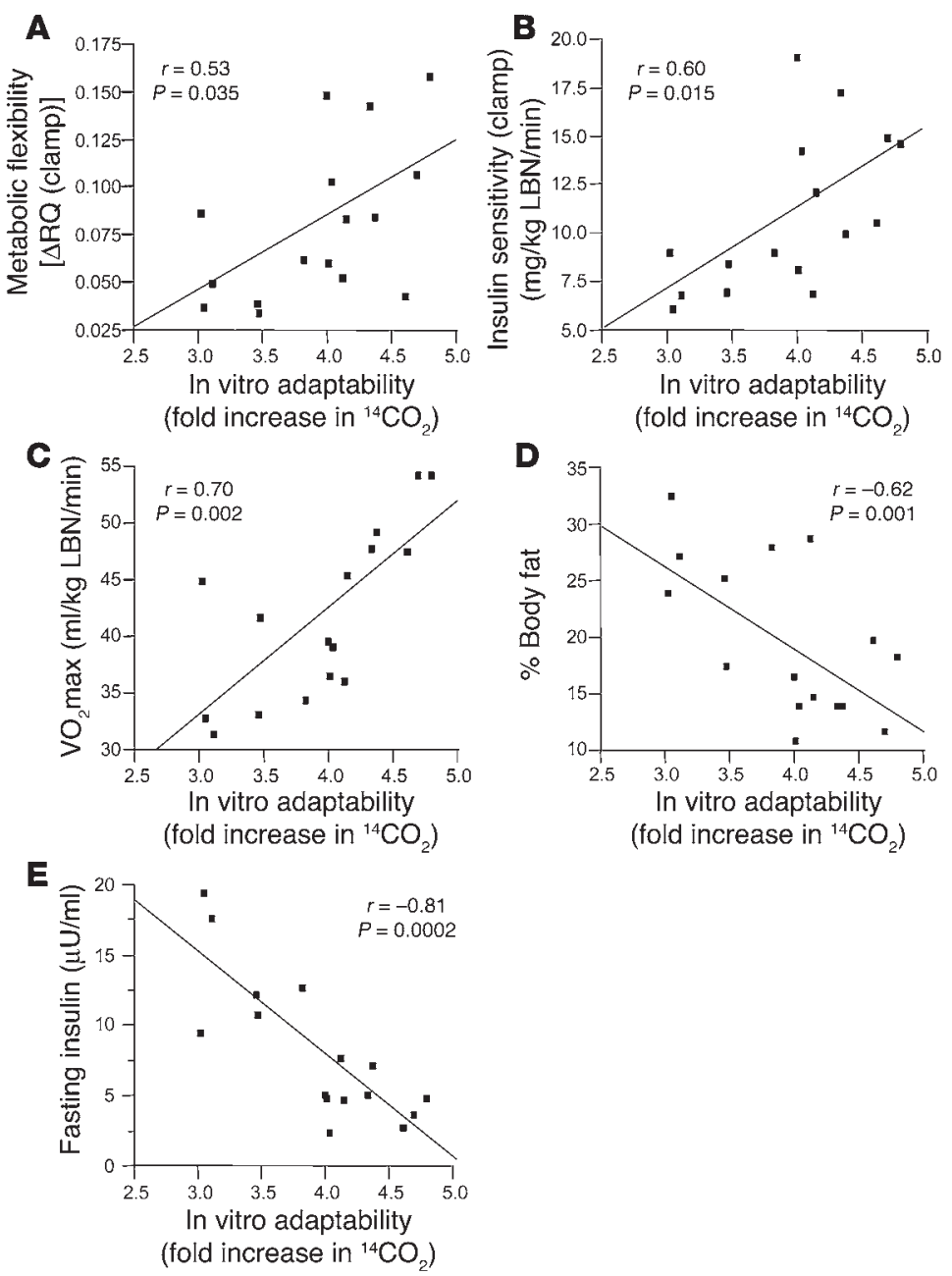

with a BMI greater than $40 \mathrm{~kg} / \mathrm{m}^{2}$ and in type 2 diabetics (43-45). Our results suggest that differences in the dynamics of FOx in young, healthy individuals mirror the metabolic characteristics of the donor. Moreover, the change in $\mathrm{CO}_{2}$ production correlates better with clinical phenotypes than does the change in production of intermediate metabolites of FOx. This might be due to the fact that $\mathrm{CO}_{2}$ represents the end product of FOx, while intermediate metabolites (ASPs) can be reused for lipid synthesis (64).

The relationship between in vitro metabolic switching and in vivo insulin responsiveness points out the importance of FOx for an insulin-sensitive phenotype. Intrinsic defects in metabolic switching of skeletal muscle might participate in the generation of a "lipotoxic milieu" in the muscle cell $(13,14)$ that acts in concert with environmental factors, such as a HFD, to favor the accumulation of lipids and lipid intermediates implicated in insulin resistance (34-37). According to our observations, skeletal muscle does not appear to be an innocent bystander but rather an active participant in the pathogenesis of obesity and insulin resistance.

Furthermore, our results from cells incubated in an insulin-free medium demonstrate that insulin is not needed for the expression of the metabolic switching phenotype in muscle cells. It is possible that a decrease in FOx observed in the fasting diabetic individual, as exemplified by an increase in fasting RQ, might be due to a defect in the ability of a muscle cell to oxidize fat or due to the sensitivity

\section{Figure 3}

In vitro adaptability is an intrinsic characteristic of muscle cells. The capacity of muscle cells to increase FOx (measured by ${ }^{14} \mathrm{CO}_{2}$ production) in the presence of high palmitate concentration (fold increase in ${ }^{14} \mathrm{CO}_{2}={ }^{14} \mathrm{CO}_{2}$ at $100 \mu \mathrm{M}$ palmitate $/{ }^{14} \mathrm{CO}_{2}$ at $0 \mu \mathrm{M}$ palmitate) in vitro is correlated with flexible clinical phenotype $[\Delta R Q$ (clamp)] (A); insulin-sensitive clinical phenotype (IS is represented by the glucose disposal rate, measured by clamp) (B); $\mathrm{VO}_{2} \max$ in vivo (C); percent body fat, measured by dual energy $\mathrm{X}$-ray absorptiometry (D); and fasting insulin levels on a standard diet (E). Muscle cells from 16 individuals were grown and differentiated into myotubes in 24-well plates. Myotubes were preincubated in glucose- and serum-free medium and incubated for 3 hours with $1 \mu \mathrm{Ci} / \mathrm{ml}{ }^{14} \mathrm{C}$-palmitate, in the presence or absence of $100 \mu \mathrm{m}$ cold palmitate. After incubation, levels of ${ }^{14} \mathrm{CO}_{2}$ and ${ }^{14} \mathrm{C}$-intermediate metabolites of FOx were determined. Assays were performed in duplicate, and data were normalized to protein content. Data were adjusted for basal ${ }^{14} \mathrm{CO}_{2}$ production $\left({ }^{14} \mathrm{CO}_{2}\right.$ production at $1 \mu \mathrm{Ci} / \mathrm{ml}$ labeled palmitate and $0 \mu \mathrm{M}$ cold palmitate).

of the cell to increasing extracellular glucose, which causes it to turn off FOx. Leg balance studies showing decreased FOx in diabetes are consistent with our findings $(1,5,23)$.

There are 2 major possible explanations for the variability in metabolic switching. First, the relatively strong in vitroin vivo relationships suggest that metabolic switching has a genetic origin. A second possibility is that myotubes in culture were previously programmed by their in vivo environment. Exposure to unknown in situ conditions might cause imprinting of the genome, imposing long-lasting effects on the cellular phenotype, as was demonstrated in rats and humans (65-67). The possible influence of diet or other environmental factors on the genome in the course of life also cannot be excluded and will require further study. In either case - whether influenced by genetic or epigenetic factors - skeletal muscle appears to play a decisive role in the handling of a nutritional challenge, such as a HFD.

In conclusion, we found that metabolic switching, representing dynamic changes in FOx in skeletal muscle cells, was correlated with clinical phenotypes of cells' donors. Myotubes retained the characteristics of the donor. This indicates that metabolic switching is, at least to a certain extent, an intrinsic characteristic of skeletal muscle, which suggests that defects in metabolic switching might be one of the primary events in the development of obesity and insulin resistance.

\section{Methods}

Study population and design. The "Mechanisms of Interindividual Differences in the Adaptation to High-Fat Diets" protocol (also known as the ADAPT study) is a cross-sectional, short-term interventional study designed to examine interindividual differences in FOx when subjects are placed on an isoenergetic HFD for 3 days. The protocol was approved by the institutional review board of the Pennington Biomedical Research center. All volunteers gave written, informed consent. Healthy young men and women, aged $18-29$ years, with a BMI of $20-35 \mathrm{~kg} / \mathrm{m}^{2}$ underwent complete physical examination, routine medical laboratory tests, and anthropometry. After completing the screening process, participants presented to the Pennington inpatient unit on day -4 and ate a weight-maintaining diet consisting of $35 \%$ fat, $16 \%$ protein, and $49 \%$ carbohydrate. On 


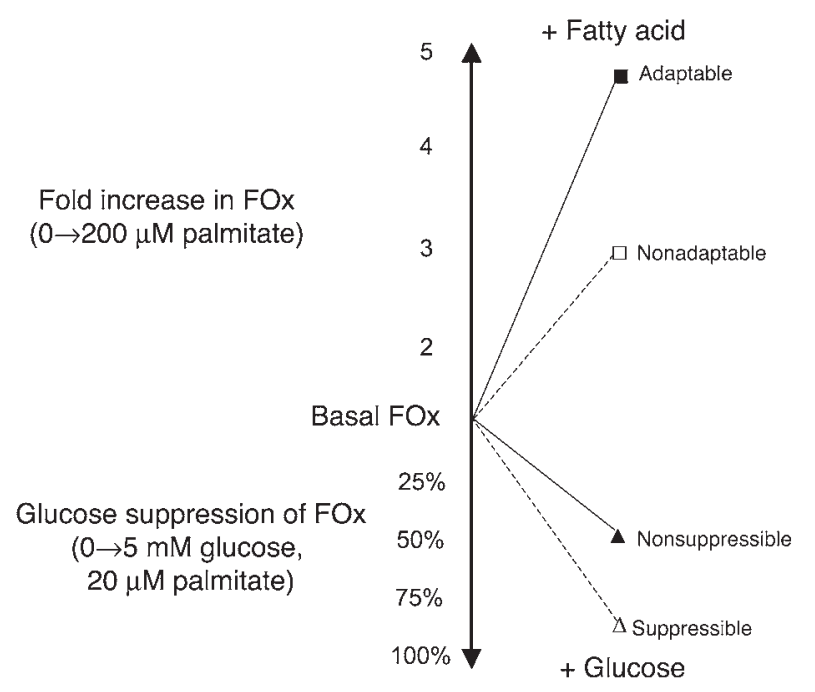

- Subjects with higher insulin sensitivity, metabolic flexibility, and lower \% body fat -.-.-- Subjects with lower insulin sensitivity, metabolic inflexibility, and highter $\%$ body fat

\section{Figure 4}

Model of in vitro metabolic switching. Glucose suppressibility: In insulinfree medium, adaptable cells are able to maintain a relatively high rate of FOx in the presence of glucose compared with nonadaptable cells. According to our studies, in vivo insulin responsiveness decreases with increasing in vitro suppressibility, which indicates greater glucose suppression of FOx in insulin resistance and metabolic inflexibility. Metabolic adaptability: Adaptable cells possess a higher capacity to increase FOx when exposed to a high palmitate concentration compared with nonadaptable cells. According to our studies, in vivo insulin responsiveness increases with increasing in vitro adaptability, which indicates a greater capacity to increase FOx in subjects with higher IS.

day -3 , a clamp was performed. On day 1 , participants entered a whole room calorimeter and continued on the same weight-maintaining $35 \%$ fat diet. Total daily energy expenditure, FOx, protein oxidation, and carbohydrate oxidation were measured at energy balance (mean energy balance over 4 days, $30.7 \pm 79.2 \mathrm{kcal} / 24 \mathrm{~h}$ [mean $\pm \mathrm{SD}]$ ).

Clamp. IS was measured by clamp (68) prior to the start of the HFD. Subjects were asked to refrain from vigorous physical activity for 48 hours before the clamp. After an overnight fast, insulin $\left(80 \mathrm{mU} / \mathrm{m}^{2} / \mathrm{min}\right)$ and $20 \%$ glucose (to maintain plasma glucose at $90 \mathrm{mg} / \mathrm{dL}$ ) were administered for 3 hours. Glucose and insulin were measured in 3 independent blood plasma samples 5 minutes apart at baseline and again at steady-state insulin and glucose levels after approximately 2 hours. Glucose disposal rate, a parameter of IS, was adjusted for kilograms of LBM. Fasting RQ, steady-state RQ (clamp RQ), and substrate oxidations for fat and carbohydrate were calculated from $\mathrm{O}_{2}$ consumption and $\mathrm{CO}_{2}$ production $\left[\mathrm{RQ}=\mathrm{CO}_{2}\right.$ produced $/ \mathrm{O}_{2}$ consumed], measured before the clamp (fasting values) and during the steady state of the clamp by indirect calorimetry. An insulin-stimulated change in RQ, the parameter of metabolic flexibility, quantifies the readiness of metabolism to switch from fasting FOx to insulin-stimulated glucose oxidation $[\Delta R Q($ clamp $)=R Q$ at the end of the insulin-stimulated glucose infusion - fasting RQ] (14).

$\mathrm{VO}_{2} \max . \mathrm{VO}_{2} \max$ was determined by a progressive treadmill test to exhaustion in accordance with the recommendations described by the American College of Sports Medicine (69). The volume of $\mathrm{O}_{2}\left(\mathrm{VO}_{2}\right)$ and $\mathrm{CO}_{2}$ was measured continuously using a metabolic cart (Vmax29 series; SensorMedics). Heart rate was monitored continuously using a portable heart rate monitor (Polar S-610; Polar Beat).
Body composition. Body fat mass and LBM were measured on a Hologic Dual Energy X-Ray Absorptiometer in the fan beam mode (QDR 2000; Hologic Inc.). Coefficients of variation for the measurement of the LBM, fat mass, and percentage of body fat were $0.8 \%, 1.6 \%$, and $1.7 \%$, respectively. Visceral fat was measured by CT scanning using a high-speed CT scanner under an established protocol (70).

Respiratory chamber. Twenty-four-hour energy expenditure and RQ were determined in a whole room respiratory calorimeter as previously described (71). $\mathrm{O}_{2}$ and $\mathrm{CO}_{2}$ levels were measured using a Magnos $4 \mathrm{G}$ magneto-pneumatic oxygen analyzer (Hartmann and Braun). Energy expenditure and substrate oxidations were calculated from $\mathrm{O}_{2}$ consumption, $\mathrm{CO}_{2}$ production, and 24-hour urinary nitrogen excretion using equations established by Acheson et al. (72). Coefficients of variation for total daily energy expenditure and RQ are $2 \%$ and $16 \%$, respectively. RQ were adjusted for energy balance as described previously (71).

Skeletal muscle cell culture. Muscle biopsy was performed on day -2 (standard diet) and day 5 (third day on the HFD). Cultures were obtained from samples from the second biopsy. Samples of vastus lateralis, weighing 60-100 mg, were obtained by muscle biopsy using the Bergstrom technique (73). Satellite cells (quiescent mononuclear muscle cells) were isolated by trypsin digestion, preplated on an uncoated petri dish for an hour to remove fibroblasts, and subsequently transferred to T-25 collagen-coated flasks in DMEM supplemented with $9 \%$ FBS and growth factors (human epidermal growth factor, BSA, dexamethasone, gentamycin, fungizone, fetuin) as previously described $(74,75)$. Cells were passaged once, harvested, and frozen at approximately $80 \%$ confluence. When ready for an experiment, cells were thawed and seeded into 24 -well plates at a density of $40 \times 10^{3}$ cells per well. Cells were grown at $37^{\circ} \mathrm{C}$ in a humidified atmosphere of $5 \% \mathrm{CO}_{2}$. Differentiation of myoblasts into myotubes was initiated at approximately $90 \%$ confluence, by switching to $\alpha$-MEM with antibiotics, $2 \%$ FBS, and fetuin. The medium was changed every other day. Measurement of FOx was performed after 5 days of differentiation, when about $70-80 \%$ of mononuclear myoblasts had fused to form multinuclear elongated myotubes. Occasional spontaneous contractions were observed.

FOx assay. Cells were preincubated with a glucose- and serum-free medium for 90 minutes, and this was followed by a 3-hour incubation with $\left[1-{ }^{14} \mathrm{C}\right]$-labeled palmitate $(1 \mu \mathrm{Ci} / \mathrm{ml}$; NEN Life Science Products) and nonlabeled (cold) palmitate, with or without glucose. Palmitate was coupled to a fatty acid-free BSA in a molar ratio of 5:1.

In vitro suppressibility assay. The in vitro suppressibility assay was designed to measure the ability of myotubes to maintain FOx in the presence of glucose, in insulin-free medium (that is, fuel preference of metabolic switching). In preliminary experiments $(n=7)$, we incubated cells with labeled palmitate and $20 \mu \mathrm{M}$ cold palmitate with $0,0.5,1.5$, and $5 \mathrm{mM}$ glucose to determine the relationship between FOx and glucose concentration. For the rest of experiments, only maximal FOx (without glucose) and suppressed FOx (with $5 \mathrm{mM}$ glucose) were determined.

In vitro adaptability assay. The in vitro adaptability assay focused on the capacity of myotubes to increase FOx in response to an acute exposure to a high palmitate concentration. In preliminary experiments $(n=8)$, we incubated cells with labeled palmitate and 0, 20, 50, and $100 \mu \mathrm{M}$ nonlabeled (cold) palmitate, in the absence of glucose and insulin, to determine the relationship between FOx and palmitate concentration. For other experiments, only basal FOx (at $1 \mu \mathrm{Ci} / \mathrm{ml}, 0 \mu \mathrm{M}$ cold palmitate) and FOx (at 100 $\mu \mathrm{M}$ palmitate) were determined. Following incubation, ${ }^{14} \mathrm{CO}_{2}$ and ${ }^{14} \mathrm{C}$-ASPs were measured according to the method of Muoio et al. (75), with some modifications. Briefly, assayed medium was transferred into a 48-well trapping plate. The plate was clamped and sealed, and perchloric acid was injected through the holes in the lid into the medium, which drove $\mathrm{CO}_{2}$ through the tunnel into an adjacent well, where it was trapped in $1 \mathrm{M} \mathrm{NaOH}$. Fol- 
lowing trapping, media was spun twice for the measurement of ${ }^{14} \mathrm{C}$-ASPs. Aliquots of $\mathrm{NaOH}$ and medium were transferred into scintillation vials, and radioactivity was measured on a multipurpose scintillation counter (LS 6500; Beckman Coulter). Cells were washed twice and harvested into $0.3 \mathrm{ml}$ of $0.05 \% \mathrm{SDS}$ for subsequent protein measurement. All assays were performed in duplicate, and data were normalized to protein content.

Calculations. Absolute rates of FOx were adjusted for specific activity to account for a dilution of ${ }^{14} \mathrm{C}$-palmitate with unlabeled palmitate. TOx was calculated as the sum of the $\mathrm{CO}_{2}$ and ASPs produced. In vitro suppressibility was calculated as percent suppression of $\mathrm{FOx}[\%$ suppression $=$ $(1-$ [FOx at $5 \mathrm{mM}$ glucose / FOx at $0 \mathrm{mM}$ glucose] $) \times 100]$. In vitro adaptability was calculated as fold increase of FOX [fold increase $=$ FOx at 100 $\mu \mathrm{M}$ cold palmitate / FOx at $0 \mu \mathrm{M}$ cold palmitate]. Calculations were performed separately for $\mathrm{CO}_{2}$ and TOx.

Statistical analysis. All clinical data were entered into the Pennington Biomedical Research Center database, extracted, combined with the in vitro fatty oxidation assay data, and analyzed using JMP software version 5.0.1a (SAS Institute Inc.). Correlations were performed in a pairwise fashion using the Pearson product moment correlation. To account for the effect of basal FOx on the fold increase in FOx, the semi-partial correlation was computed to examine the relations between fold increase FOx and clinical characteristics (BMI, $\mathrm{VO}_{2} \max$, fasting insulin, etc.), while basal FOx production was held as a constant. The semi-partial correlation coefficient was calculated in SAS (version 8.2; SAS Institute) using the PROC REG subroutine. All values are presented in figures and tables as sample (raw) mean \pm SE. The type II error rate was set a priori at $P<0.05$.

\section{Acknowledgments}

This work was supported by US Department of Agriculture grant 200334323 14010. We thank Eric Ravussin and Ruth Loos for helpful advice and Lauren Sparks and Iwona Bogacka for help and support. Our great appreciation goes to Jozef Ukropec for his advice, support, and inspiring discussions. We also thank the volunteers who participated in this study.

Received for publication December 27, 2004, and accepted in revised form April 26, 2005.

Address correspondence to: Steven R. Smith, Pennington Biomedical Research Center, 6400 Perkins Road, Baton Rouge, Louisiana 70808, USA. Phone: (225) 763-3028; Fax: (225) 763-0274; E-mail: smithsr@pbrc.edu.
1. Colberg, S.R., Simoneau, J.A., Thaete, F.L., and Kelley, D.E. 1995. Skeletal muscle utilization of free fatty acids in women with visceral obesity. J. Clin. Invest. 95:1846-1853.

2. Simoneau, J.A., and Kelley, D.E. 1997. Altered glycolytic and oxidative capacities of skeletal muscle contribute to insulin resistance in NIDDM. J. Appl. Physiol. 83:166-171.

3. He, J., Watkins, S., and Kelley, D.E. 2001. Skeletal muscle lipid content and oxidative enzyme activity in relation to muscle fiber type in type 2 diabetes and obesity. Diabetes. 50:817-823.

4. Doucet, E., Tremblay, A., Simoneau, J.A., and Joanisse, D.R. 2003. Skeletal muscle enzymes as predictors of 24-h energy metabolism in reducedobese persons. Am. J. Clin. Nutr. 78:430-435.

5. Kelley, D.E., Goodpaster, B., Wing, R.R., and Simoneau, J.A. 1999. Skeletal muscle fatty acid metabolism in association with insulin resistance, obesity, and weight loss. Am. J. Physiol. 277:E1130-E1141.

6. Raben, A., Mygind, E., and Astrup, A. 1998. Lower activity of oxidative key enzymes and smaller fiber areas in skeletal muscle of postobese women. Am J. Physiol. 275:E487-E494.

7. Ranneries, C., et al. 1998. Fat metabolism in formerly obese women. Am. J. Physiol. 274:E155-E161.

8. Zurlo, F., et al. 1990. Low ratio of fat to carbohydrate oxidation as predictor of weight gain: study of 24-h RQ. Am. J. Physiol. 259:E650-E657.

9. Ravussin, E., and Swinburn, B.A. 1993. Metabolic predictors of obesity: cross-sectional versus longitudinal data. Int. J. Obes. Relat. Metab. Disord. 17(Suppl. 3):S28-S31; discussion S41-S42.

10. Meigs, J.B., Muller, D.C., Nathan, D.M., Blake, D.R., and Andres, R. 2003. The natural history of progression from normal glucose tolerance to type 2 diabetes in the Baltimore Longitudinal Study of Aging. Diabetes. 52:1475-1484.

11. Marra, M., Scalfi, L., Covino, A., Esposito-Del Puente, A., and Contaldo, F. 1998. Fasting respiratory quotient as a predictor of weight changes in non-obese women. Int. J. Obes. Relat. Metab. Disord. 22:601-603

12. Bruce, C.R., et al. 2003. Muscle oxidative capacity is a better predictor of insulin sensitivity than lipid status. J. Clin. Endocrinol. Metab. 88:5444-5451.

13. Storlien, L., Oakes, N.D., and Kelley, D.E. 2004. Metabolic flexibility. Proc. Nutr. Soc. 63:363-368.

14. Kelley, D.E., and Mandarino, L.J. 2000. Fuel selection in human skeletal muscle in insulin resistance: a reexamination. Diabetes. 49:677-683.

15. Smith, S.R., et al. 2000. Fat and carbohydrate balances during adaptation to a high-fat. Am. J. Clin. Nutr. 71:450-457.

16. Verboeket-van de Venne, W.P., Westerterp, K.R., and ten Hoor, F. 1994. Substrate utilization in man: effects of dietary fat and carbohydrate. Metabolism. 43:152-156.

17. Astrup, A., Buemann, B., Christensen, N.J., and Toubro, S. 1994. Failure to increase lipid oxidation in response to increasing dietary fat content in formerly obese women. Am. J. Physiol. 266:E592-E599.

18. Buemann, B., Toubro, S., and Astrup, A. 1998. Substrate oxidation and thyroid hormone response to the introduction of a high fat diet in formerly obese women. Int. J. Obes. Relat. Metab. Disord. 22:869-877.

19. Thomas, C.D., et al. 1992. Nutrient balance and energy expenditure during ad libitum feeding of high-fat and high-carbohydrate diets in humans. Am. J. Clin. Nutr. 55:934-942.

20. Heitmann, B.L., Lissner, L., Sorensen, T.I., and Bengtsson, C. 1995. Dietary fat intake and weight gain in women genetically predisposed for obesity. Am. J. Clin. Nutr. 61:1213-1217.

21. Mandarino, L.J., Consoli, A., Jain, A., and Kelley, D.E. 1996. Interaction of carbohydrate and fat fuels in human skeletal muscle: impact of obesity and NIDDM. Am. J. Physiol. 270:E463-E470.

22. Kelley, D.E., and Simoneau, J.A. 1994. Impaired free fatty acid utilization by skeletal muscle in noninsulin-dependent diabetes mellitus. J. Clin. Invest. 94:2349-2356.

23. Kelley, D., Mokan, M., and Veneman, T. 1994. Impaired postprandial glucose utilization in noninsulin-dependent diabetes mellitus. Metabolism. 43:1549-1557.

24. Wolfe, B.M., Klein, S., Peters, E.J., Schmidt, B.F., and Wolfe, R.R. 1988. Effect of elevated free fatty acids on glucose oxidation in normal humans. Metabolism. 37:323-329.

25. Sidossis, L.S., and Wolfe, R.R. 1996. Glucose and insulin-induced inhibition of fatty acid oxidation: the glucose-fatty acid cycle reversed. Am. J. Physiol. 270:E733-E738.

26. Winder, W.W., Arogyasami, J., Elayan, I.M., and Cartmill, D. 1990. Time course of exercise-induced decline in malonyl-CoA in different muscle types. Am. J. Physiol. 259:E266-E271.

27. Yamauchi, T., et al. 2001. The fat-derived hormone adiponectin reverses insulin resistance associated with both lipoatrophy and obesity. Nat. Med. 7:941-946.

28. Weyer, C., et al. 2001. Hypoadiponectinemia in obesity and type 2 diabetes: close association with insulin resistance and hyperinsulinemia. J. Clin. Endocrinol. Metab. 86:1930-1935.

29. Havel, P.J. 2002. Control of energy homeostasis and insulin action by adipocyte hormones: leptin, acylation stimulating protein, and adiponectin. Curr. Opin. Lipidol. 13:51-59.

30. Boden, G., et al. 1991. Effects of fat on insulinstimulated carbohydrate metabolism in normal men. J. Clin. Invest. 88:960-966.

31. Kleiber, H., et al. 1992. Interaction of lipid and carbohydrate metabolism after infusions of lipids or of lipid lowering agents: lack of a direct relationship between free fatty acid concentrations and glucose disposal. Diabete. Metab. 18:84-90.

32. Kelley, D.E., Mokan, M., Simoneau, J.A., and Mandarino, L.J. 1993. Interaction between glucose and free fatty acid metabolism in human skeletal muscle. J. Clin. Invest. 92:91-98.

33. Roden, M., et al. 1996. Mechanism of free fatty acid-induced insulin resistance in humans. J. Clin. Invest. 97:2859-2865.

34. Adams, J.M., 2nd, et al. 2004. Ceramide content is increased in skeletal muscle from obese insulinresistant humans. Diabetes. 53:25-31.

35. Schmitz-Peiffer, C. 2002. Protein kinase C and lipid-induced insulin resistance in skeletal muscle. Ann. N. Y. Acad. Sci. 967:146-157.

36. Shulman, G.I. 2000. Cellular mechanisms of insulin resistance. J. Clin. Invest. 106:171-176.

37. Kraegen, E.W., Cooney, G.J., Ye, J., and Thompson, A.L. 2001. Triglycerides, fatty acids and insulin resistance - hyperinsulinemia [review]. Exp. Clin. Endocrinol. Diabetes. 109:S516-S526.

38. Unger, R.H. 2003. Lipid overload and overflow: metabolic trauma and the metabolic syndrome. Trends Endocrinol. Metab. 14:398-403.

39. Henry, R.R., Ciaraldi, T.P., Mudaliar, S., Abrams, L., and Nikoulina, S.E. 1996. Acquired defects of glycogen synthase activity in cultured human skeletal muscle cells: influence of high glucose and insulin levels. Diabetes. 45:400-407.

40. Henry, R.R., Abrams, L., Nikoulina, S., and Ciaraldi, T.P. 1995. Insulin action and glucose metabolism in nondiabetic control and NIDDM subjects. Comparison using human skeletal muscle cell cultures. 
Diabetes. 44:936-946.

41. Mott, D.M., et al. 2000. Palmitate oxidation rate and action on glycogen synthase in myoblasts from insulin-resistant subjects. Am. J. Physiol. Endocrinol. Metab. 279:E561-E569.

42. Gaster, M., and Beck-Nielsen, H. 2004. The reduced insulin-mediated glucose oxidation in skeletal muscle from type 2 diabetic subjects may be of genetic origin - evidence from cultured myotubes. Biochim. Biophys. Acta. 1690:85-91.

43. Kim, J.Y., Hickner, R.C., Cortright, R.L., Dohm, G.L., and Houmard, J.A. 2000. Lipid oxidation is reduced in obese human skeletal muscle. Am.J. Physiol. Endocrinol. Metab. 279:E1039-E1044.

44. Hulver, M.W., et al. 2003. Skeletal muscle lipid metabolism with obesity. Am. J. Physiol. Endocrinol. Metab. 284:E741-E747.

45. Gaster, M., Rustan, A.C., Aas, V., and Beck-Nielsen, H. 2004. Reduced lipid oxidation in skeletal muscle from type 2 diabetic subjects may be of genetic origin: evidence from cultured myotubes. Diabetes. 53:542-548.

46. Abate, N., Garg, A., Peshock, R.M., Stray-Gundersen, J., and Grundy, S.M. 1995. Relationships of generalized and regional adiposity to insulin sensitivity in men. J. Clin. Invest. 96:88-98.

47. Bavenholm, P.N., et al. 2003. Insulin resistance in type 2 diabetes: association with truncal obesity, impaired fitness, and atypical malonyl coenzyme A regulation. J. Clin. Endocrinol. Metab. 88:82-87.

48. Toth, M.J., Sites, C.K., Cefalu, W.T., Matthews, D.E., and Poehlman, E.T. 2001. Determinants of insulin-stimulated glucose disposal in middle-aged, premenopausal women. Am. J. Physiol. Endocrinol. Metab. 281:E113-E121.

49. Oakes, N.D., Ljung, B., and Camejo, G. 2002. Correction of dysfunctional fatty acid metabolism using peroxisome proliferator activated receptor gamma agonists. J. R. Soc. Med. 95(Suppl. 42):33-38.

50. Smith, S.R., et al. 2000. Concurrent physical activity increases fat oxidation during the shift to a high-fat diet. Am. J. Clin. Nutr. 72:131-138.

51. Abdel-aleem, S., et al. 1996. Regulation of fatty acid oxidation by acetyl-CoA generated from glucose utilization in isolated myocytes. J. Mol. Cell. Cardiol. 28:825-833.

52. Saddik, M., Gamble,J., Witters, L.A., and Lopaschuk,
G.D. 1993. Acetyl-CoA carboxylase regulation of fatty acid oxidation in the heart. J. Biol. Chem. 268:25836-25845.

53. Stuart, C.A., et al. 2001. Altered GLUT1 and GLUT3 gene expression and subcellular redistribution of GLUT4: protein in muscle from patients with acanthosis nigricans and severe insulin resistance. Metabolism. 50:771-777.

54. Ciaraldi, T.P., Abrams, L., Nikoulina, S., Mudaliar, S., and Henry, R.R. 1995. Glucose transport in cultured human skeletal muscle cells. Regulation by insulin and glucose in nondiabetic and non-insulin-dependent diabetes mellitus subjects. J. Clin. Invest. 96:2820-2827.

55. Kirkwood, S.P., Zurlo, F., Larson, K., and Ravussin, E. 1991. Muscle mitochondrial morphology, body composition, and energy expenditure in sedentary individuals. Am. J. Physiol. 260:E89-E94.

56. Kelley, D.E., He, J., Menshikova, E.V., and Ritov, V.B. 2002. Dysfunction of mitochondria in human skeletal muscle in type 2 diabetes. Diabetes. 51:2944-2950.

57. Blaak, E.E., and Saris, W.H. 2002. Substrate oxidation, obesity and exercise training. Best Pract. Res. Clin. Endocrinol. Metab. 16:667-678.

58. Blaak, E.E., and Wagenmakers, A.J. 2002. The fate of [U-(13)C]palmitate extracted by skeletal muscle in subjects with type 2 diabetes and control subjects. Diabetes. 51:784-789.

59. Winder, W.W., Arogyasami, J., Barton, R.J., Elayan, I.M., and Vehrs, P.R. 1989. Muscle malonylCoA decreases during exercise. J. Appl. Physiol. 67:2230-2233.

60. Ruderman, N., and Prentki, M. 2004. AMP kinase and malonyl-CoA: targets for therapy of the metabolic syndrome. Nat. Rev. Drug Discov. 3:340-351.

61. Bavenholm, P.N., Pigon, J., Saha, A.K., Ruderman, N.B., and Efendic, S. 2000. Fatty acid oxidation and the regulation of malonyl-CoA in human muscle. Diabetes. 49:1078-1083.

62. Debard, C., et al. 2004. Expression of key genes of fatty acid oxidation, including adiponectin receptors, in skeletal muscle of type 2 diabetic patients. Diabetologia. 47:917-925.

63. Abu-Elheiga, L., Oh, W., Kordari, P., and Wakil, S.J. 2003. Acetyl-CoA carboxylase 2 mutant mice are protected against obesity and diabetes induced by high-fat/high-carbohydrate diets. Proc. Natl. Acad.
Sci. U. S. A. 100:10207-10212.

64. Bartlett, K., and Eaton, S. 2004. Mitochondrial beta-oxidation. Eur. J. Biochem. 271:462-469.

65. Taylor, P.D., et al. 2005. Impaired glucose homeostasis and mitochondrial abnormalities in offspring of rats fed a fat-rich diet in pregnancy. Am.J. Physiol. Regul. Integr. Comp. Physiol. 288:R134-R139.

66. Roberts, R.M. 2004. Impact of maternal diet on reproductive outcome: foreword. Biol. Reprod. 71:1045.

67. Fleming, T.P., et al. 2004. The embryo and its future. Biol, Reprod. 71:1046-1054.

68. DeFronzo, R.A., Tobin, J.D., and Andres, R. 1979. Glucose clamp technique: a method for quantifying insulin secretion and resistance. Am. J. Physiol. 237:E214-E223.

69. Tanaka, H., Monahan, K.D., and Seals, D.R. 2001. Age-predicted maximal heart rate revisited. J. Am. Coll. Cardiol. 37:153-156.

70. Smith, S.R., et al. 2001. Contributions of total body fat, abdominal subcutaneous adipose tissue compartments, and visceral adipose tissue to the metabolic complications of obesity. Metabolism. 50:425-435.

71. Nguyen, T., de Jonge, L., Smith, S.R., and Bray, G.A. 2003. Chamber for indirect calorimetry with accurate measurement and time discrimination of metabolic plateaus of over $20 \mathrm{~min}$. Med. Biol. Eng. Comput. 41:572-578.

72. Acheson, K.J., et al. 1984. Nutritional influences on lipogenesis and thermogenesis after a carbohydrate meal. Am. J. Physiol. 246:E62-E70.

73. Ravussin, E., Lillioja, S., Anderson, T.E., Christin, L., and Bogardus, C. 1986. Determinants of 24hour energy expenditure in man. Methods and results using a respiratory chamber. J. Clin. Invest. 78:1568-1578.

74. Bergstrom, J. 1975. Percutaneous needle biopsy of skeletal muscle in physiological and clinical research. Scand. J. Clin. Lab. Invest. 35:609-616.

75. Muoio, D.M., et al. 2002. Peroxisome proliferator-activated receptor-alpha regulates fatty acid utilization in primary human skeletal muscle cells. Diabetes. 51:901-909.

76. Blau, H.M., and Webster, C. 1981. Isolation and characterization of human muscle cells. Proc. Natl. Acad. Sci. U. S. A. 78:5623-5627. 